\title{
Empiema y efusión pleural en niños
}

\author{
M. Francisca Arancibia G., Luis E. Vega-Briceño, M. Ester Pizarro G., Dahiana Pulgar B., Nils Holmgren P., \\ Pablo Bertrand N., José I. Rodríguez C. e Ignacio Sánchez D.
}

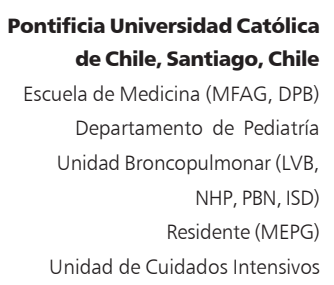

(JIRC)

Recibido: 25 abril 2007 Aceptado: 3 septiembre 2007

Correspondencia a: Luis Enrique Vega-Briceño levega@puc.cl

\section{Empyema and pleural efussion in children}

Introduction: Pleural empyema (PE) is a serious complication of community-acquired pneumonia (CAP). Objectives: To describe the clinical profile of hospitalized patients with $\mathrm{PE}$ in the pediatric ward of the Catholic University Hospital between 2000-2005. Patients y methods: Retrospectively, all pediatric admission due to CAP and pleural effusion (86 children) were identified. In 59 (70\%) children $\geq 1$ thoracocentesis were performed. We considered PE as the presence in the pleural effusion of pus, and/or a positive gram strain and/ or positive culture, and/or a $\mathrm{pH}<7.10$. Children with effusions not meeting any criteria were used as controls. Results: Twenty four PE and 25 controls were identified, with a global mean age of 2.9 years (range: 8 months to 14.3 years); $78 \%$ were $<5$ years, with a significant difference between PE and controls [1.6 vs 3.3 years $(\mathrm{p}=0.01)]$. The mean duration of symptoms in PE patients before admission was 7 days (range: $2-21)$, and the most frequent symptoms were fever (100\%) and cough (96\%). In 15/24 cases a microorganism was identified being Streptococcus pneumoniae $(\mathrm{n}=9)$ the most common. In 48 patients management was conservative and in 4 surgical procedures were required. The mean duration of hospitalization was significantly higher in the PE group vs controls group: 15 (range: 5-38) vs 9 days (range 3-16) $(\mathrm{p}<0.01)$. A chest tube was inserted in 83\% of children with EP compared with $36 \%$ in the control group $(\mathrm{p}=0.002)$. There were no difference in number of days of oxygen use [6 vs $4.5(\mathrm{p}=0.36)]$ or number of chest tubes per child $[3 v s 2.5(\mathrm{p}=0.29)]$. No deaths were reported. Conclusion: PE in children represented an acute respiratory event associated with more prolonged hospitalization especially at younger ages; the majority of cases did not require surgical intervention.

Key words: Pleural effusion, empyema, complicated pneumonia, children.

Palabras claves: Efusión pleural, empiema, neumonía complicada, niños.

\section{Introducción}

$\mathrm{E}$ 1 empiema pleural (EP) es una complicación grave que afecta hasta $28 \%$ de los pacientes pediátricos hospitalizados por neumonía adquirida en la comunidad (NAC) $)^{1}$. Si bien el EP es una condición común a muchos hospitales del mundo, su manejo y tratamiento óptimo, son aún motivo de gran controversia ${ }^{2-6}$; estos incluyen antibioterapia y toracocentesis $^{7-9}$, tubo de drenaje pleural ${ }^{10,11}$, fibrinolíticos ${ }^{12-14}$, cirugía toracoscópica video asistida ${ }^{15-17}$ y toracotomía abierta ${ }^{7,18}$; siendo la mayoría de ellos practicados de manera secuencial y escalonada. Afortunadamente, y de manera independiente del tratamiento elegido como primario, la mayoría de estos niños responde bien y normaliza de manera completa sus pruebas de función pulmonar, luego de 18 meses $^{8}$.

El EP traduce una condición fisiopatológica dinámica caracterizada por la presencia de fiebre alta y persistente, a pesar del inicio precoz de antibioterapia. Según algunos investigadores, el tratamiento médico o conservador (definido como el uso de antimicrobianos y tubo de drenaje) se asocia a hospitalizaciones prolongadas $^{5,19}$ y frecuentemente a fracasos que requieren una intervención quirúrgica de rescate $^{19}$, por lo que muchos cirujanos pediátricos, sugieren la intervención quirúrgica precoz ${ }^{20-22}$. Un reciente meta-análisis publicado, que incluyó ocho estudios clínicos bien diseñados, reveló una tasa de fracaso casi 11 veces más en los pacientes que recibieron tratamiento médico vs el grupo quirúrgico primario ${ }^{19}$; sin embargo, más de $76 \%$ de los pacientes que recibieron tratamiento médico conservador no requirieron intervención quirúrgica alguna durante su hospitalización.

En nuestro medio, Paz y cols, describieron 81 pacientes con NAC y efusión pleural, hospitalizados en el periodo 1985-2000, no existiendo publicaciones nacionales que describan el perfil clínico o epidemiológico de esta grave complicación respiratoria después de ello ${ }^{23}$. Ya que el manejo del EP en nuestra institución es eminentemente médico (conservador) y sólo en forma ocasional se practica la cirugía toracoscópica video asistida (CTVA o VATS, por sus siglas en inglés) o toracotomía abierta, fue nuestro objetivo describir las 
principales características clínicas y de laboratorio de los niños hospitalizados por EP, brindando una actualización en torno a esta controversia y una eventual aproximación de manejo médico-quirúrgico.

\section{Pacientes y Métodos}

Se identificaron de manera retrospectiva, todos los pacientes pediátricos hospitalizados por NAC y efusión pleural durante el período 2000-2005 en el Servicio de Pediatría del Hospital Clínico de la Pontificia Universidad Católica. Se revisó la ficha médica registrando las condiciones crónicas de base, factores de riesgo, síntomas y signos previos a la admisión, motivo de la hospitalización y su evolución, exámenes de laboratorio (incluyendo análisis de líquido pleural y sangre) complicaciones, tratamientos indicados y condición final al alta.

Criterios de inclusión: pacientes con NAC y efusión pleural en quienes se practicó al menos una toracocentesis efectiva (entendida como la obtención de líquido pleural) para su estudio.

Criterios de exclusión: pacientes que desarrollaron colecciones pleurales post-quirúrgicas.

Definiciones. Fiebre: presencia de temperatura corporal $\geq 37,5{ }^{\circ} \mathrm{C}$ axilar o $\geq 38^{\circ} \mathrm{C}$ rectal; reaparición de fiebre: presencia de este síntoma luego de haber permanecido al menos 48 horas sin fiebre; hipoxemia: saturación arterial de oxígeno $\left(\mathrm{SaO}_{2}\right) \leq 90 \%$; EP presencia de pus, tinción de Gram con bacterias, cultivo positivo o $\mathrm{pH}<7,10$ en el líquido pleural; controles: todos aquéllos que no cumplían criterios de EP (considerado como efusión para-neumónica); condición crónica de base: presencia de prematurez, alergia, sibilancias recurrentes, antecedentes de episodio previo de neumonía, enfermedad neurológica severa o genopatía; niño sano: ausencia de las condiciones antes mencionadas.

Análisis estadístico. Se consideró todas las variables numéricas y categóricas, siendo las variables numéricas con distribución normal expresadas como media \pm desviación estándar o en su defecto como medianas y rangos. Para establecer asociaciones numéricas entre dos variables se empleó la prueba $t$ de Student (variables numéricas con distribución normal), MannWhitney (análisis no paramétrico) o $\chi^{2}$ (variables categóricas), considerando diferencias significativas aquellas con $\mathrm{p}<0,05$ empleando para ello el paquete estadístico STATCAL 2000 y Statplus 2005 para windows.

\section{Resultados}

Se identificaron 4.356 hospitalizaciones por alguna causa respiratoria en nuestro servicio; $86(2 \%)$ de ellas correspondieron a NAC con efusión pleural, realizándose al menos una toracocentesis en 59 (70\%) pacientes. De ellos, en tres casos no se obtuvo líquido pleural y en siete no se encontró la ficha médica, por lo que finalmente se revisaron 49 (83\%) fichas médicas, siendo incluidas para el análisis 24 EP y 25 controles. El diagnóstico de EP se estableció por cultivo positivo en 11/24 (Streptococcus pneumoniae en 9 casos), tinción de Gram con microrganismos en 9/24, presencia de pus en $7 / 24$ y $\mathrm{pH}<7,10$ en $7 / 24$. En tres casos la tinción de Gram no mostró bacterias aunque el cultivo fue positivo (todos fueron $S$. pneumoniae), en cuatro la tinción de Gram mostró diplococos grampositivos siendo el cultivo informado negativo, en cinco la toracocentesis mostró pus pero la tinción de Gram y el cultivo fueron negativos. El rendimiento bacteriológico del líquido en esta serie fue de $63 \%(15 / 24)$.

Hallazgos clínicos. La Tabla 1 muestra las principales características clínicas de ambos grupos. La edad promedio fue 2,9 años (rango: 8 meses-14,3 años); 53 y $78 \%$ tenían bajo 3 y 5 años de edad, respectivamente. Existió una diferencia significativa entre los grupos EP y control [1,6 (rango: 8 meses-14,3 años) versus 3,3 (rango: 1,2-12,8) años, respectivamente $(p=0,01)]$. Ochenta y ocho por ciento de los niños del grupo EP tenían bajo 2 años de edad, a diferencia del grupo control $(8 \%)(\mathrm{p}=0,002)$; más aún, los lactantes presentaron hospitalizaciones más prolongadas que los pacientes sobre 2 años de edad [16 días (rango: 5-38) versus 9,5 días (3-36), respectivamente $(p=0,02)]$ asociadas a $\mathrm{pH}$ de líquido pleural significativamente inferior que sus controles $[7,03$ vs 7,35 $(\mathrm{p}=0,008)]$. No se pudo realizar análisis estadístico entre los pacientes bajo 2 años de edad con respecto a su condición de EP o control, debido al escaso número de sujetos con esta edad en el grupo control $(n=2)$.

La Figura 1 muestra los signos y síntomas más frecuentes reportados. Diecinueve niños eran sanos previamente, presentando 30 alguna condición crónica de base: 25 sibilancias recurrentes, 6 algún episodio de neumonía bacteriana, 4 prematurez y 4 alguna enfermedad neurológica (parálisis cerebral 1 caso y epilepsia 3 casos). Ocho tenían más de una condición crónica de base simultánea.

La estadía promedio de hospitalización global fue 10 días (rango: 3-38), siendo significativamente superior en el grupo $\operatorname{EP}(\mathrm{p}=0,003)$ (Figura 2). Los 13 niños derivados de otra institución mostraron una estadía hospitalaria más prolongada [17 (rango: 7-38) versus 


\begin{tabular}{|c|c|c|c|c|c|c|c|}
\hline \multirow[b]{2}{*}{ Edad (años)* } & \multirow{2}{*}{\multicolumn{2}{|c|}{$\begin{array}{ll}\text { Todos } \\
\\
(\mathbf{n}=\mathbf{4 9})\end{array}$}} & \multirow{2}{*}{\multicolumn{2}{|c|}{$\begin{array}{l}\text { Empiema pleural } \\
\text { (n = 24) } \\
1,6(8 m-14,3 a)\end{array}$}} & \multicolumn{2}{|c|}{$\begin{array}{l}\text { Controles } \\
(n=25)\end{array}$} & \multirow{2}{*}{$\begin{array}{c}\mathbf{p} \\
0,012\end{array}$} \\
\hline & & & & & 3,3 & $a-12,8 a)$ & \\
\hline Bajo 2 años de edad n (\%) & 17 & (35) & 15 & $(62,5)$ & 2 & (8) & 0,0002 \\
\hline Mujeres n (\%) & 29 & $(59)$ & 17 & $(71)$ & 12 & $(48)$ & 0,18 \\
\hline Condición crónica de base n (\%) & 30 & $(61)$ & 17 & $(71)$ & 13 & $(52)$ & 0,29 \\
\hline Duración de síntomas pre-ingreso (días)* & 7 & $(2-21)$ & 7 & $(4-21)$ & 6 & $(2-20)$ & 0,11 \\
\hline Duración de fiebre pre-ingreso (días)* & 5 & $(1-18)$ & 6 & $(1-14)$ & 5 & $(1-18)$ & 0,21 \\
\hline Antimicrobianos pre-ingreso n (\%) & 22 & $(45)$ & 8 & $(33)$ & 14 & $(56)$ & 0,24 \\
\hline AINES pre-ingreso $\mathrm{n}(\%)$ & 28 & $(57)$ & 11 & $(46)$ & 17 & $(68)$ & 0,19 \\
\hline Duración de hospitalización (días)* & 10 & $(3-38)$ & 15 & $(5-38)$ & 9 & $(3-16)$ & 0,0003 \\
\hline Duración de fiebre en hospital (días)* & 3,6 & $(0-11,9)$ & 4,2 & $(0-11,9)$ & 3,2 & $(0-11,3)$ & 0,73 \\
\hline Cambio de antimicrobiano n (\%) & 30 & $(61,2)$ & 20 & $(83)$ & 10 & $(40)$ & 0,005 \\
\hline Oxígeno (días)* & 5 & $(0,25-20)$ & 6 & $(1-20)$ & 4,5 & $(0,25-12)$ & 0,36 \\
\hline Uso de drenaje pleural n (\%) & 29 & $(59)$ & 20 & $(83)$ & 9 & $(36)$ & 0,002 \\
\hline Duración de drenaje pleural (días)* & 3 & $(2-20)$ & 3 & $(2-20)$ & 3 & $(2-6)$ & 0,29 \\
\hline Volumen de drenaje pleural $(\mathrm{ml})^{*}$ & 228 & $(32-1.060)$ & 220 & $(32-1.031)$ & 228 & $(45-1.060)$ & 0,66 \\
\hline Cirugía n (\%) & 5 & $(10)$ & 4 & $(17)$ & 1 & $(4)$ & 0,32 \\
\hline Ingreso a UPC n (\%) & 25 & (53) & 16 & $(67)$ & 9 & (36) & 0,06 \\
\hline Pacientes derivados n (\%) & 13 & $(27)$ & 8 & (33) & 5 & $(20)$ & 0,46 \\
\hline
\end{tabular}

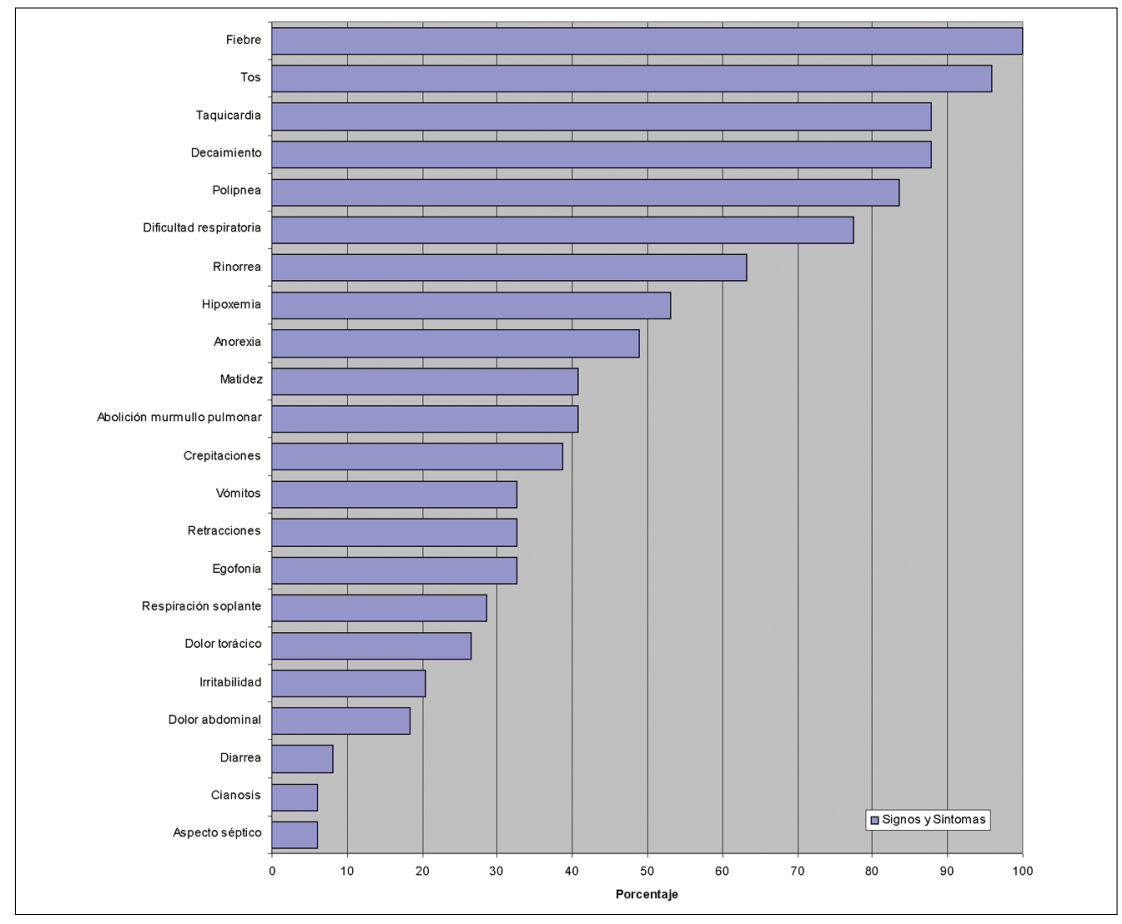

Figura 1. Características clínicas asociadas a neumonía adquirida en la comunidad y efusión pleural en 49 pacientes pediátricos.
10 días (rango: $3-21)$, respectivamente $(\mathrm{p}=0,006)]$. El año 2003 concentró $30 \%$ de las hospitalizaciones, ocurriendo $40 \%$ durante los meses de mayo, junio y julio.

Los pacientes con EP por $S$. pneumoniae no mostraron diferencias clínicas respecto al resto. La temperatura promedio máxima alcanzada fue $39,5^{\circ} \mathrm{C}$ (rango: $38-41{ }^{\circ} \mathrm{C}$ ), sólo dos pacientes no desarrollaron fiebre durante la hospitalización.

Laboratorio. Al ingreso, el recuento de leucocitos promedio fue $17.000 / \mathrm{mm}^{3}$ (rango: $2.300-34.300 / \mathrm{mm}^{3}$ ) mostrando desviación a izquierda el $65 \%$. No existió diferencia significativa entre las variables hematológicas (serie roja y blanca) evaluadas en ambos grupos. La PCR promedio global al ingreso fue de $58,4 \mathrm{mg} / \mathrm{dl}$ existiendo marcadas diferencias entre ambos grupos (EP: 87,5 vs controles: 32,0; $<<0,001$ ). Se realizó gases arteriales al ingreso en 11 pacientes ( 7 grupo EP y 4 control), mostrando en promedio $\mathrm{pH} 7,34( \pm 0,14), \mathrm{PaO}_{2}$ $88 \mathrm{mmHg}( \pm 74,2), \mathrm{PaCO}_{2} 38,1( \pm 8,27)$ sin existir diferencia significativa entre ambos grupos.

Se solicitó hemocultivos en 27 oportunidades, resultando 4 positivos (3 en grupo EP). Streptococcus pneumoniae (multisensible) fue el agente más frecuentemente aislado (2 casos), Haemophilus influenzae no 
tipificable y Streptococcus pyogenes se recuperaron en un caso, cada cual.

La Tabla 2 muestra las principales características bioquímicas y citológicas de los líquidos pleurales analizados. Se observó predominio polimorfonuclear en la fórmula diferencial del líquido pleural en más de $80 \%$ de los pacientes.

Tratamientos y complicaciones. Todos los niños recibieron manejo antimicrobiano ev (penicilina 21/49, cefalosporinas de $3^{\circ}$ generación $5 / 49$, cloxacilina + penicilina $4 / 49$ y cloxacilina + cefalosporina de $3^{\circ}$ generación 15/49); en 30 de ellos se efectuó algún cambio durante la hospitalización, ampliando la cobertura en 21 y disminuyéndola en 9; siendo las principales razones: deterioro clínico y/o persistencia de la fiebre (14/ $30)$, adecuación según estudio de susceptibilidad in vitro $(6 / 29)$, buena respuesta clínica (4/30), presencia de cavitaciones pulmonares (2/30), desarrollo de fiebre intra-hospitalaria $(2 / 30)$ y erupción cutánea alérgica (1/30). Durante la internación, 44 niños requirieron oxígeno.

El uso de tubo de drenaje pleural fue instalado con mayor frecuencia en el grupo EP (86\%) y aunque no existió diferencia en el número de días de drenaje, éste se asoció a una mayor frecuencia de hospitalización al día diez (Figura 3). Las principales razones de ingreso a la Unidad de Paciente Crítico (UPC) fueron: alto requerimiento de oxígeno $(8 / 25)$, post-cirugía $(5 / 25)$ y monitorización post-instalación de drenaje pleural en el resto, mostrando una evolución clínica posterior similar a los pacientes de sala. En dos pacientes del grupo control fue necesaria ventilación no invasora por agotamiento respiratorio.

En 4/49 se requirió alguna intervención quirúrgica de rescate (3 CTVA y 1 toracotomía), siendo practicado en promedio al sexto día (rango: 4-9) principalmente por persistencia de fiebre asociada a loculaciones en la efusión. La intervención quirúrgica de rescate fue más frecuente en el grupo EP (3 CTVA y 1 toracotomía). Aunque no existió diferencia significativa (en términos clínicos ni laboratorio) entre los pacientes con

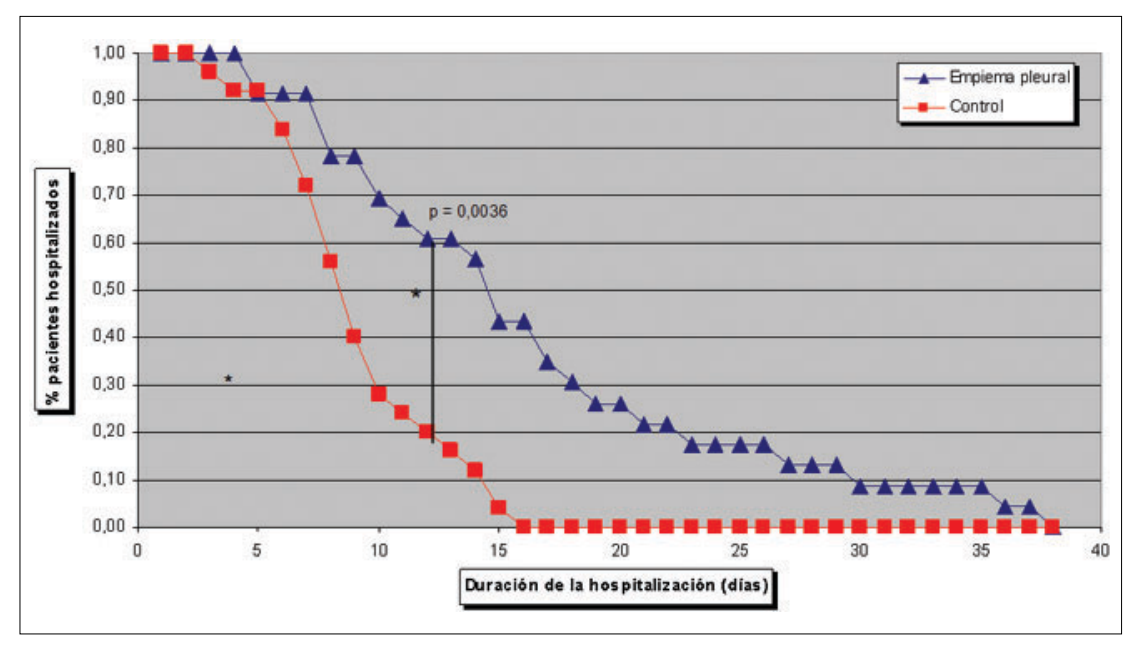

Figura 2. Duración de la hospitalización en pacientes pediátricos con empiema pleural y con efusión para-neumónica (controles).

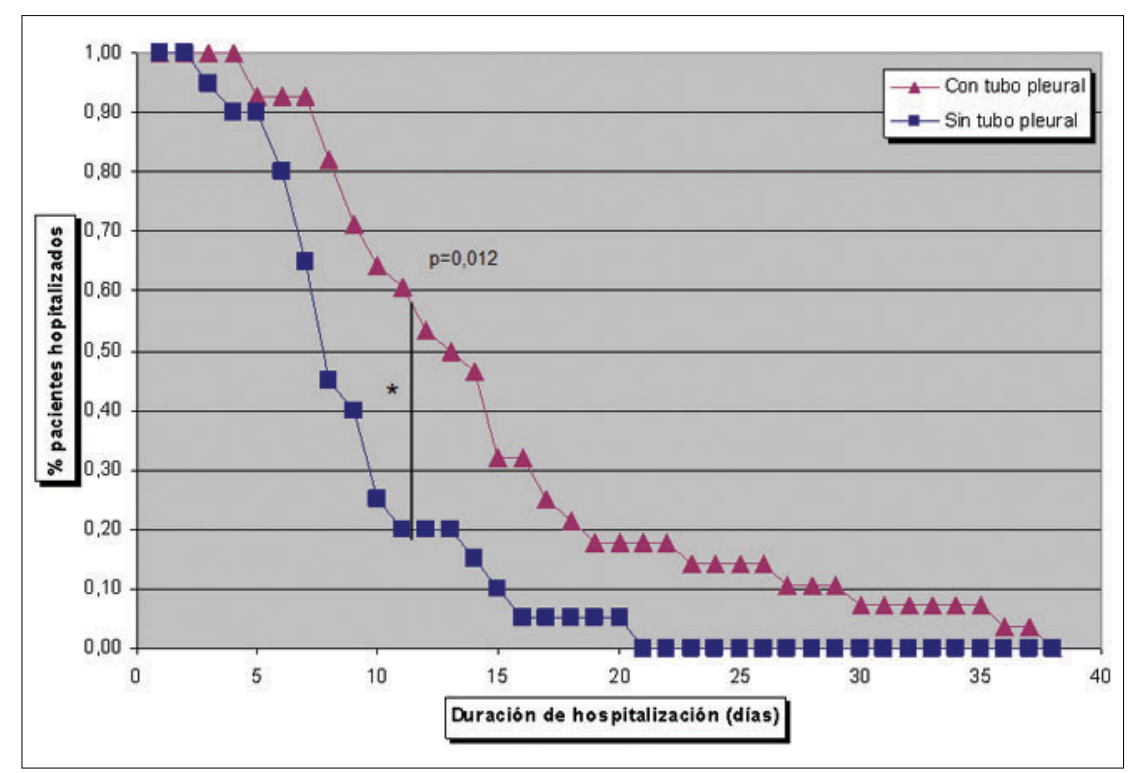

Figura 3. Duración de la hospitalización en pacientes pediátricos con empiema pleural, con y sin instalación de tubo pleural.

\begin{tabular}{|c|c|c|c|c|c|c|c|}
\hline \multirow{2}{*}{$\begin{array}{l}\text { Valores } \\
\mathrm{pH}\end{array}$} & \multicolumn{2}{|c|}{$\begin{array}{l}\text { Todos } \\
(n=49)\end{array}$} & \multicolumn{2}{|c|}{$\begin{array}{l}\text { Empiema pleural } \\
\qquad(n=24)\end{array}$} & \multicolumn{2}{|c|}{$\begin{array}{l}\text { Controles } \\
(n=25)\end{array}$} & \multirow{2}{*}{$\begin{array}{r}\mathbf{p} \\
0,94\end{array}$} \\
\hline & 7,26 & $(6,09-8,0)$ & 7,13 & $(6,09-8,0)$ & 7,33 & $(7,0-7,52)$ & \\
\hline Glucosa (mg/dl) & 52,5 & $(0-133)$ & 15,5 & $(0-130)$ & 70,5 & $(11-133)$ & 0,71 \\
\hline Proteínas (g/dl) & 4 & $(0,20-5,7)$ & 3,95 & $(0,20-5,4)$ & 4,05 & $(2,6-5,7)$ & 0,70 \\
\hline LDH (UI/I) & 1.620 & $(104-7,910)$ & 2.645 & $(104-7.910)$ & 1.396 & $(196-6.153)$ & 0,028 \\
\hline Leucocitos $\left(\mathrm{mm}^{3}\right)$ & 6.700 & $(20-81,200)$ & 8.400 & $(20-72.400)$ & 5.000 & $(500-81.200)$ & 0,01 \\
\hline
\end{tabular}




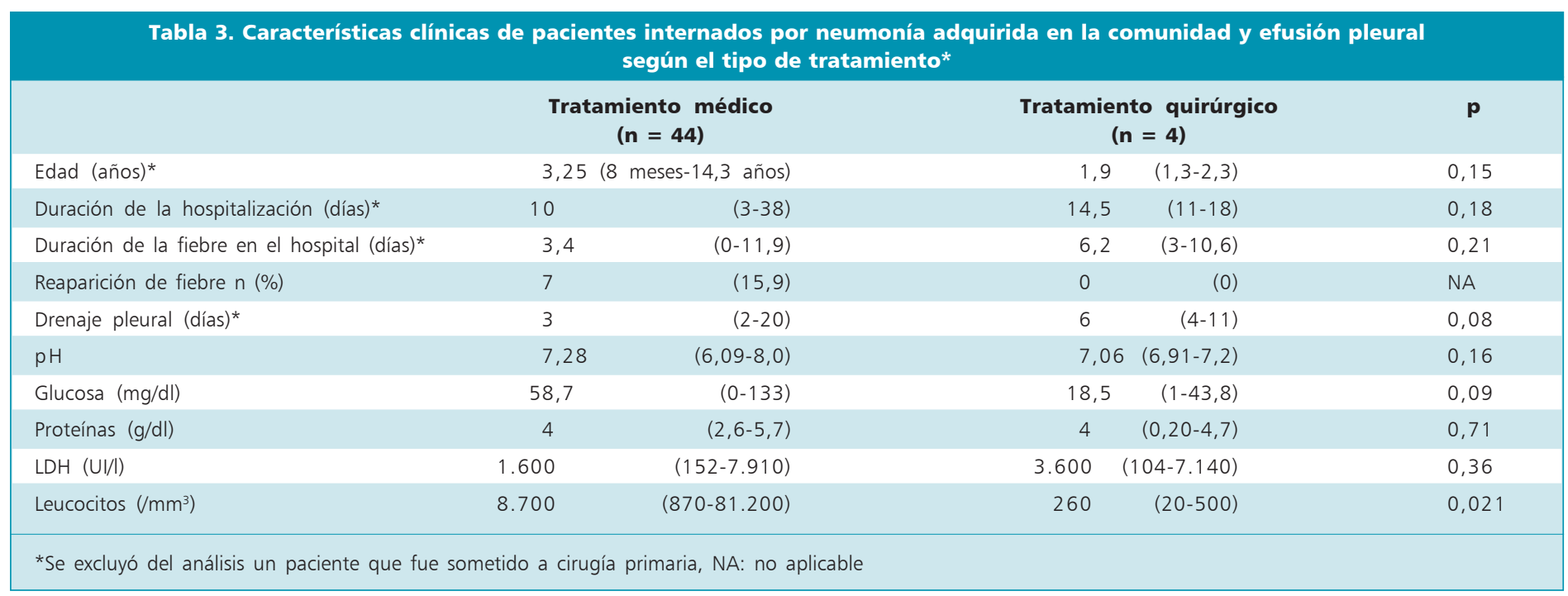

tratamiento médico o quirúrgico, se observó reaparición de fiebre con mayor frecuencia en 7 pacientes que recibieron tratamiento médico (Tabla 3). En sólo un caso se practicó CTVA primaria (primeras 24 horas del ingreso) en una lactante de 16 meses con EP y loculaciones, siendo retirado el tubo de drenaje al tercer día y permaneciendo en el hospital durante 5 días.

En esta serie no se registró fallecidos.

\section{Discusión}

Esta serie clínica retrospectiva mostró que la hospitalización por EP en pacientes pediátricos fue una condición respiratoria aguda que afectó preferentemente a los niños bajo 5 años de edad; si bien la mayoría de ellos no recibió intervención quirúrgica primaria o de rescate, la estadía hospitalaria promedio fue prolongada, especialmente en los lactantes.

El rendimiento bacteriológico en el grupo EP fue alto, siendo S. pneumoniae el agente más frecuente reportado. Aunque el número de días de drenaje pleural no fue significativamente diferente, los pacientes con EP fueron sometidos a este procedimiento con mayor frecuencia que el grupo control (efusión para-neumónica).

La presentación clínica del EP en pediatría ha mostrado un cambio en las últimas tres décadas, asociada a diversas intervenciones de manejo y control ${ }^{7,20-28}$. Streptococcus pneumoniae es hoy en día el agente más frecuentemente aislado como lo demuestran diversos estudios ${ }^{20,28}$; algunas series, hechas en países subdesarrollados, han revelado un rendimiento bacte- riológico que oscila entre 48 y $82 \%{ }^{7}$. A pesar del elevado uso de antimicrobianos previos al ingreso, observamos un rendimiento de $63 \%$. En este estudio no se identificó ningún caso de $S$. aureus, a diferencia de lo descrito en otras series ${ }^{11,25,26}$.

No encontramos diferencia en el número de días de hospitalización entre los pacientes que recibieron tratamiento conservador versus quirúrgico; sin embargo, el número de sujetos en el grupo con cirugía fue muy reducido y en sólo 4 de ellos la conducta quirúrgica fue de rescate. En tan sólo un caso se practicó CTVA como terapia primaria, paciente que cursó con una estadía hospitalaria corta y el retiro precoz del tubo pleural. Nuestros hallazgos confirman que la hospitalización asociada a EP en niños fue significativamente superior en número promedio de días de hospitalización, a otras condiciones pediátricas agudas, siendo éste un factor que se asocia a elevados costos de tratamiento ${ }^{2}$, ausentismo escolar y laboral de los padres y mayor riesgo de infecciones nosocomiales.

Pese al elevado número de publicaciones en torno a este tema, aún existe controversia -tanto en el medio nacional como internacional- en torno al tratamiento primario adecuado del EP en niños ${ }^{2-6}$, por lo que se requieren a la brevedad estudios prospectivos para responder muchas preguntas. A pesar de ello, en nuestro centro la mayoría de niños recibió un tratamiento médico conservador y de manera interesante, la tasa de éxito fue de $92 \%$; pues sólo 4 sujetos requirieron una cirugía de rescate por persistencia de la fiebre a pesar del cambio de esquema antimicrobiano asociado a loculaciones en todos los casos. Algunos investigadores más conservadores sostienen considerar los 
riesgos de ingresar un paciente con EP a pabellón, por lo que desaconsejan esta práctica.

Si bien $43 \%$ de los pacientes inició tratamiento con penicilina, se produjo un ajuste terapéutico en casi $60 \%$ de ellos, principalmente por una evolución clínica inadecuada o según la susceptibilidad in vitro. Creemos que existe una indicación abusiva de antimicrobianos en esta condición respiratoria, aunque el diseño por nosotros empleado no permite establecer mayores afirmaciones. A nuestro entender -luego de una revisión bibliográfica extensa- no existe un consenso (o criterio único) en cuanto al número de días que debe recibir antibioterapia un paciente pediátrico con $\mathrm{EP}^{7,16}$; en nuestra institución, se acostumbra mantenerlo endovenoso por al menos 3 a 5 días después de terminada la fiebre. A pesar de ello, el número de días de hospitalización del grupo EP fue 15 días y no 28 como en otras series ${ }^{11}$. Es posible que la introducción de CTVA primaria como opción terapéutica resolutiva en nuestro centro, reduzca considerablemente el número de días de hospitalización y de drenajes pleurales; sin embargo, los índices de costo-beneficio así como la seguridad de esta práctica, necesitan aún ser eva- luadas para establecer recomendaciones basadas en evidencia científica.

En la mayoría de los 86 pacientes hospitalizados por NAC y efusión pleural, sólo se practicó una toracocentesis y en un reducido número de ellos se requirió un tubo de drenaje pleural, siendo esta intervención más frecuente en el grupo EP. La colocación de tubo, no sólo se asocia a dolor en el paciente, sino que aumenta discretamente el número de días de hospitalización, aunque en esta serie no observamos diferencias estadísticamente significativas entre ambos grupos.

En cuanto al volumen de drenaje para retirar el tubo ( sin considerar parámetros clínicos como fiebre o estado general), no existe un único consenso. El espacio pleural en los pacientes con NAC y colección pleural es drenado cuando existe evidencia demostrada de EP o el volumen es abundante y su evacuación aliviará la mecánica respiratoria.

En conclusión, pese al sesgo de registro -inherente al diseño del trabajo- esta serie reveló que la mayoría de los pacientes no recibió intervención quirúrgica alguna, mostrando una tasa de éxito mayor al 90\% y

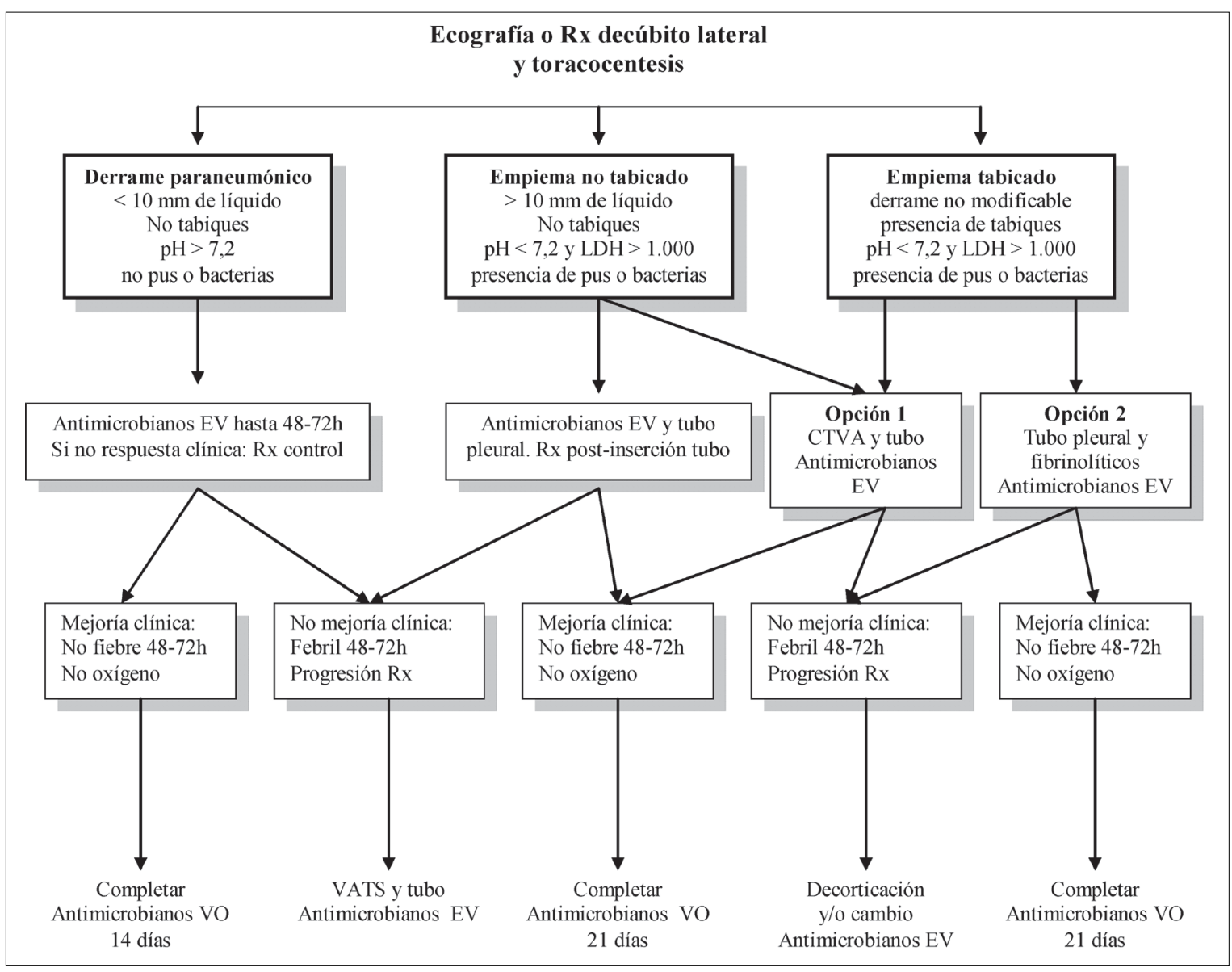

Anexo 1. Propuesta de manejo del empiema y efusión pleural en niños. CTVA: cirugía torácica video-asistida; VO: vía oral; EV: endovenoso; Rx: radiografía de tórax. Tomado y modificado de Holmgren et al. Pleuroneumonía. En: Enfoque clínico de las enfermedades respiratorias del niño. página 291; Ediciones: Pontificia Universidad Católica, 2007. 
una estadía hospitalaria promedio prolongada. Si bien su óptima terapia es aún motivo de controversia, la literatura actual -mayormente extranjera- sugiere una intervención quirúrgica precoz.

Presentamos una propuesta de manejo (Anexo 1). Si bien la resolución quirúrgica del EP complicado es la opción terapéutica más apropiada en algunos suje$\operatorname{tos}^{23,29}$, hasta la fecha, en nuestro centro se toman conductas preferentemente conservadoras en la mayoría de los pacientes. Se requieren estudios multicéntricos, prospectivos, bien diseñados, que evalúen nuevas alternativas terapéuticas para el manejo de esta grave complicación respiratoria y permitan establecer guías clínicas fundamentadas.

\section{Resumen}

Introducción: El empiema pleural (EP) es una complicación grave de la neumonía adquirida en la comunidad (NAC). Objetivos: Describir las características de los pacientes hospitalizados por EP en el Servicio de Pediatría del Hospital de la Universidad Católica durante el período 2000-2005. Se identificaron 86 hospitalizaciones por NAC con efusión pleural, practicándose en $59(70 \%)$, al menos una toracocentesis. Se consideró EP a la presencia de pus, tinción de Gram con bacterias, cultivo positivo o $\mathrm{pH}<7,10$ en el líquido pleural, siendo las efusiones para-neumónicas los controles. Resultados: Se analizaron $24 \mathrm{EP}$ y 25 controles [promedio 2,9 años (rango: 8 meses - 14,3 años)], 78\% de edad inferior a 5 años con diferencia entre los grupos EP y controles [1,6 vs 3,3 años, respectivamente $(\mathrm{p}=0,01)]$. El promedio global (días) de síntomas previo al ingreso en los EP fue 7 (rango: 2-21), siendo los más frecuentes fiebre $(100 \%)$ y tos $(96 \%)$. Se identificó algún microorganismo en 15/24 EP, Streptococcus pneumoniae fue el más frecuente (n: 9). En 48 niños, el manejo inicial fue conservador, requiriéndose cuatro rescates quirúrgicos luego del cuarto día. El promedio (días) de hospitalización fue significativamente superior en el grupo EP $v s$ controles [15 (rango: 5-38) vs 9 (rango: 3-16) $(\mathrm{p}<0,01)]$. Requirieron drenaje pleural $83 \%$ del grupo EP y $36 \%$ de los controles $(p=0,002)$. No hubo diferencia en el número de días de empleo de oxígeno [6 vs 4,5 $(\mathrm{p}=0,36)]$ o drenaje pleural [3 vs 2,5 $(\mathrm{p}=0,29)]$. No se registraron fallecidos. Conclusión: El EP en niños fue una condición respiratoria aguda que se asoció a estadías hospitalarias prolongadas, especialmente en los de menor edad, no requiriéndose, en la mayoría, una intervención quirúrgica de rescate.

\section{Referencias}

1.- Byington C L, Spencer L Y, Johnson T A, et al. An epidemiological investigation of a sustained high rate of pediatric parapneumonic empyema: risk factors and microbiological associations. Clin Infect Dis 2002; 34: 434-40.

2.- Coote N. Surgical versus non-surgical management of pleural empyema. Cochrane Database Syst Rev 2002; 2: CD001956.

3.- Gates RL, Caniano DA, Hayes JR, Arca MJ. Does VATS provide optimal treatment of empyema in children? A systematic review. J Pediatr Surg 2004; 39: 381-6.

4.- Doski J J, Lou D, Hicks B A, Megison S M, Sánchez P, Contidor M, et al. Management of parapneumonic collections in infants and children. J Pediatr Surg 2000; 35: 265-8. Discussion: 269-70.

5.- Hilliard T N, Henderson A J, Langton Hewer S C. Management of parapneumonic effusion and empyema. Arch Dis Child 2003; 88: 915-7.

6.- Jaffe A, Cohen G. Thoracic empyema. Arch Dis Child 2003; 88: 839-41.

7.- Baranwal A K, Singh M, Marwaha R K, Kumar L. Empyema thoracis: a 10-year comparative review of hospitalised children from South Asia. Arch Dis Child 2003; 88: 1009-14.

8.- Gocmen A, Kiper N, Toppare, Ozcelik U, Cengizlier R, Cetinkaya F. Conservative treatment of empyema in children. Respiration 1993; 60: 182-5.

9.- Shoseyov D, Bibi H, Shatzberg G, Klar A, Akerman J, Hurvitz H, Maayan C. Short-term course and outcome of treatments of pleural empyema in pediatric patients: repeated ultrasound-guided needle thoracocentesis vs chest tube drainage. Chest 2002; 121: 836-40.

10.- Gates R L, Hogan J, Weinstein S, Arca M J. Drainage, fibrinolytics, or surgery: a comparison of treatment options in pediatric empyema. J Pediatr Surg 2004; 39: 1638-42.

11.- Yilmaz E, Dogan Y, Aydinoglu A H, Gurgoze MK, Aygun D. Parapneumonic empyema in children: conservative approach. Turk J Pediatr 2002; 44: 134-8.

12.- Bouros D, Schiza S, Tzanakis S, Chalkiadakis G, Drositis J, Siafakas N. Intrapleural urokinase versus normal saline in the treatment of complicated parapneumonic effusions and empyema.
A randomized, double-blind study. Am J Respir Crit Care Med 1999; 159: 37-42.

13.- Thomson A H, Hull J, Kumar M R, Wallis C, Balfour Lynn I M. Randomised trial of intrapleural urokinase in the treatment of childhood empyema. Thorax 2002; 57: 343-7.

14.- Singh M, Mathew J J, Chandra S, Katariya S, Kumar L. Randomized controlled trial of intrapleural streptokinase in empyema thoracis in children. Acta Paediatr 2004; 93: 1443-5.

15.- Subramaniam R, Joseph V T, Tan G M, Goh A, Chay O M. Experience with videoassisted thoracoscopic surgery in the management of complicated pneumonia in children. J Pediatr Surg 2001; 36: 316-9.

16.- Grewal H, Jackson R J, Wagner C W, Smith S D. Early Video-assisted thoracic surgery in the management of empyema. Pediatrics 1999; 103: 63.

17.- Klena J W, Cameron B H, Langer J C, Winthrop A L, Pérez C R. Timing of videoassisted thoracoscopic debridement for pediatric empyema. J Am Coll Surg 1998; 187: 404-8.

18.- Karaman I, Erdogan D, Karaman A, Cakmak O. Comparison of closed-tube 
thoracostomy and open thoracotomy procedures in the management of thoracic empyema in childhood. Eur J Pediatr Surg 2004; 14: 250-4.

19.- Avansino J R, Goldman B, Sawin R S, Flum D R. Primary operative versus nonoperative therapy for pediatric empyema: a meta-analysis. Pediatrics 2005; 115: 1652-9.

20.- Schultz K, Fan L L, Pinsky J, Ochoa L, Smith E O, Kaplan S L, et al. The changing face of pleural empyemas in children: epidemiology and management. Pediatrics 2004; 113: 1735-40.

21.- Grewal H, Jackson R, Wagner C, Smith S. Early video-assisted thoracic surgery in the management of empyema. 1999; 103: e63.
22.- Subramanian R, Joseph VT Tan G M, Goh A, Chay O M. Experience with video-assisted thoracoscopic surgery in the management of complicated pneumonia in children. J Pediatr Surg 2001; 36: 316-9.

23.- Paz F, Céspedes P, Cuevas M, Lecorre N, Navarro H, García C, et al. Pleural efussion and complicated empyema in children. Evolution and prognostic factors. Rev Med Chile 2001; 129: 1289-96.

24.- Vega-Briceno L E, Perret C, Holmgren N, Sánchez I. Non-typable Haemophilus influenzae severe pneumonia in an infant: case report. Rev Chil Infectol 2005; 22: 8992.

25.- Ozel S K, Kazez A, Kilic M, Koseugullari A A, Yilmaz E, Aygun A D. Conservative treatment of postpneumonic empyema in children. Surg Today 2004; 34: 1002-5.

26. - Hailu S. Paediatric thoracic empyema in an Ethiopian referral hospital. East Afr Med J 2000; 77: 618-21.

27.- Shankar K R, Kenny S E, Okoye B O, Carty H M, Lloyd D A, Losty P D. Evolving experience in the management of empyema thoracis. Acta Paediatr 2000; 89: 417-20.

28.- Buckingham S C, King M D, Miller M L. Incidence and etiologies of complicated parapneumonic effusions in children, 1996-2001. Pediatr Infect Dis J 2003; 22: 499-504.

29. - Álvarez C, Zúñiga S, Corrales R, García C, Aparicio R, Sánchez I. Pleuroneumonia tabicada en niños: Diagnóstico y tratamiento quirúrgico. Rev Chil Enf Respir 1994; 10: 128-35. 